\title{
Incidence d'une technique de décontamination par tensioactifs sur la conductivité hydraulique d'un aquifère contrôlé pollué par du gazole
}

\section{Incidence of surfactant decontamination technique on the hydraulic conductivity of a controlled aquifer polluted by diesel oil}

\author{
M. Bettahar, O. Razakarisoa, F. Van Dorpe et M. Baviere
}

Volume 11, numéro 1, 1998

URI : https://id.erudit.org/iderudit/705298ar

DOI : https://doi.org/10.7202/705298ar

\section{Aller au sommaire du numéro}

\section{Éditeur(s)}

Université du Québec - INRS-Eau, Terre et Environnement (INRS-ETE)

\section{ISSN}

0992-7158 (imprimé)

1718-8598 (numérique)

Découvrir la revue

Citer cet article

Bettahar, M., Razakarisoa, O., Van Dorpe, F. \& Baviere, M. (1998). Incidence d'une technique de décontamination par tensioactifs sur la conductivité hydraulique d'un aquifère contrôlé pollué par du gazole. Revue des sciences de l'eau / Journal of Water Science, 11(1), 85-100. https://doi.org/10.7202/705298ar

\section{Résumé de l'article}

Les nappes d'eau souterraine constituent des ressources vulnérables que l'activité industrielle croissante contribue à polluer trop fréquemment. Les produits les plus manipulés se voient donc directement concernés dans les cas de pollution d'aquifères. A ce titre, les produits pétroliers, notamment ceux que l'on considère comme étant domestiques (gazole, fuel, essence, etc.) se situent au premier rang.

Dans le but de mieux aborder ce type de contamination, des études sont menées sur un site expérimental de grandes dimensions et parfaitement contrôlé, dans lequel $476 \mathrm{l}$ de gazole routier (GOR86) ont été déversés. Une première tentative de récupération du polluant, par infiltration de tensioactifs depuis la surface, démontre la capacité du mélange utilisé à mobiliser le polluant mais conduit à la réduction de la perméabilité du milieu poreux faisant ainsi apparaître une limitation des performances pour ce type de procédés de décontamination.

Des expériences au laboratoire, conduites sur des colonnes de milieu poreux, ont été entreprises pour rechercher les causes de cette perte de conductivité hydraulique. Avec le sable considéré ici, les argiles ne jouent pas un rôle prépondérant dans le colmatage. Il est démontré également que le gradient de charge, quand il est augmenté, permet d'injecter une quantité plus importante de tensioactifs sans pour autant pallier ce problème de colmatage. Enfin, il apparaît que les tensioactifs, en présence des ions calcium, peuvent s'agglomérer pour former des associations de micelles lamellaires, cylindriques et/ou mixtes, assez volumineux pour être filtrés à la surface du sol à traiter. Au bout d'un certain temps, le milieu poreux est totalement colmaté et par conséquent le débit d'infiltration de la solution devient nul. Sur le plan de la récupération du polluant, ce paramètre s'avère être particulièrement important. Sa variation influence donc fortement les quantités de polluant mobilisé.

Cette étude démontre la faisabilité du procédé. Toutefois elle souligne aussi que, sous peine de perdre de leur efficacité, les solutions de tensioactifs doivent conserver leurs caractéristiques initiales (stabilité de la taille des particules colloïdales notamment) pendant toute la durée du traitement. Dans la mesure où l'agglomération des micelles est en cause, il faudra rechercher les moyens d'obtenir une dispersion plus stable, par exemple par addition d'agents solvants. 


\title{
Incidence d'une technique de décontamination par tensioactifs sur la conductivité hydraulique d'un aquifère contrôlé pollué par du gazole
}

\author{
Influence of a surfactant decontamination technique \\ on the hydraulic conductivity of a controlled aquifer \\ polluted by diesel oil
}

\author{
M. BETTAHAR ${ }^{1}$, 0. RAZAKARISOA ${ }^{1}$, F. VAN DORPE ${ }^{1}$ \\ et M. BAVIERE2
}

Reçu le 27 juillet 1996, accepté le 17 novembre 1997*.

In case of groundwater contamination by hydrocarbon spills, one of the main problems is how to recover residual hydrocarbons trapped in the porous medium, forming a long term pollution source. In order to develop a better approach to such problems, experiments were conducted in a large experimental controlled site, called SCERES (Site Contrôlé Expérimental de Recherche pour la Réhabilitation des Eaux et des Sols) made of an impervious concrete basin ( $25 \mathrm{~m} \times 12 \mathrm{~m} \times 3 \mathrm{~m}$ ) packed with two layers of quartz sand and fitted with specific instrumentation.

This research site was experimentally polluted with diesel oil (476 litres), then the shape of the impregnation body was identified and oil saturation values were quantified thanks to a specific coring programme before and after each step of the experiments (water table fuctuations, hydraulic pumping, surfactant infiltration, etc.). The principle of the remediation technique is based on a surfactant flushing from the soil surface. This process was carried out when all of the removable diesel oil had been recovered by hydraulic pumping in the central recovery well. The surfactant infiltration allowed a displacement of the main part of the residual pollutant in the vertical zone of the impregnation body, in spite of a progressive reduction of hydraulic conductivity leading to a plugging of the porous matrix. This phenomenon has been observed by many scientists (ALLRED et BROWN, 1994 ; CELIK et al., 1982) and explained as being caused by a precipitation of the anionic surfactant in presence of calcium ions.

This decrease of hydraulic conductivity, due to surfactant infiltration and its effects, was studied in the laboratory by the implementation of experiments using columns filled with the same sand as in the SCERES basin. The results showed that the reduction of the hydraulic conductivity of the porous medium cannot be due to the precipitation of the anionic surfactants in presence of cal-

1. Institut Mécanique des Fluides de Strasbourg, Institut Franco-Allemand de Recherche sur l'Environnement, URA 854, CNRS-ULP, 23, rue du Loess, BP 20,67037 Strasbourg Cedex, France.

2. Institut Français du Pétrole, 1 et 4, avenue de Bois-Préau, BP 311, 92506 Rueil-Malmaison, France.

* Les commentaires seront reçus jusqu'au 6 novembre 1998. 
cium ions of the sand. If this were situation, all the porous medium in the column would have exhibited this loss of hydraulic conductivity. In our case, only the upstream part of the physical model was influenced by this phenomenon.

Furthermore, the sand devoid of its clay minerals has nearly the same behaviour as a natural sand, with respect to the infiltration flowrate of the surfactants. This proves that the clay minerals, present in small proportion in this matrix, are not mainly responsible for the plugging problem. The influence of the hydraulic gradient, another studied parameter, was observed and permitted to apprehend the behaviour of the surfactant solution during its infiltration through the porous medium. The increase of this parameter, even if it allows the injection of more surfactant, could not resolve the problem of the reduction of hydraulic conductivity.

Finally, all the experiments indicated that the plugging effect (cancellation of the flowrate) happened after nearly 20 hours of surfactant infiltration through the porous matrix. The time parameter seems to be important since its variation can influence the stability of the surfactant solution. The results indicated that we can infiltrate a greater volume of the solution if it has been recently prepared. In other words, this is certainly due to the time necessary for the formation of liquid crystals obtained in presence of the calcium ions in the water used for the preparation of the solutions (tap water). After nearly 20 hours these crystals should have attained a size sufficient to be retained by a filtration effect, near the soil surface. We suggest that this filtration is the major factor responsible for the reduction of hydraulic conductivity and the plugging.

The present study shows the feasibility of this process and it highlights the need to conserve the initial characteristics of the surfactants (especially the size stability of the colloidal particles) during all the treatment. Because of the agglomeration of the micelles, we have to search for means to ensure a good dispersion in the aqueous medium, for example by adding a solvent agent.

Key-words : decontamination, surfactants, hydraulic conductivity, aquifer, oil pollution.

\section{RÉSUMÉ}

Les nappes d'eau souterraine constituent des ressources vulnérables que l'activité industrielle croissante contribue à polluer trop fréquemment. Les produits les plus manipulés se voient donc directement concernés dans les cas de pollution d'aquiferes. À ce titre, les produits pétroliers, notamment ceux que l'on considère comme étant domestiques (gazole, fuel, essence, etc.) se situent au premier rang.

Dans le but de mieux aborder ce type de contamination, des études sont menées sur un site expérimental de grandes dimensions et parfaitement contrôlé, dans lequel 476 I de gazole routier (GOR86) ont été déversés. Une première tentative de récupération du polluant, par infiltration de tensioactifs depuis la surface, démontre la capacité du mélange utilisé à mobiliser le polluant mais conduit à la réduction de la perméabilité du milieu poreux faisant ainsi apparaître une limitation des performances pour ce type de procédés de décontamination.

Des expériences au laboratoire, conduites sur des colonnes de milieu poreux, ont été entreprises pour rechercher les causes de cette perte de conductivité hydraulique. Avec le sable considéré ici, les argiles ne jouent pas un rôle prépondérant dans le colmatage. Il est démontré également que le gradient de charge, quand il est augmenté, permet d'injecter une quantité plus importante de tensioactifs sans pour autant pallier ce problème de colmatage. Enfin, il apparaît que les tensioactifs, en présence des ions calcium, peuvent s'agglomérer pour former des associations de micelles lamellaires, cylindriçues et/ou 
mixtes, assez volumineux pour être filtrés à la surface du sol à traiter. Au bout d'un certain temps, le milieu poreux est totalement colmaté et par conséquent le débit d'infiltration de la solution devient nul. Sur le plan de la récupération du polluant, ce paramètre s'avère être particulièrement important. Sa variation influence donc fortement les quantités de polluant mobilisé.

Cette étude démontre la faisabilité du procédé. Toutefois elle souligne aussi que, sous peine de perdre de leur efficacité, les solutions de tensioactifs doivent conserver leurs caractéristiques initiales (stabilité de la taille des particules collöidales notamment) pendant toute la durée du traitement. Dans la mesure où l'agglomération des micelles est en cause, il faudra rechercher les moyens d'obtenir une dispersion plus stable, par exemple par addition d'agents solvants.

Mots clés : décontamination, tensioactifs, conductivité hydraulique, aquifêre, pollution, gazole.

\section{1 - INTRODUCTION}

La contamination des eaux souterraines par des produits pétroliers constitue un des problèmes majeurs qui menacent la qualité de l'environnement. Lors d'une telle altération d'aquifères alluviaux, la principale difficulté vient du fait que, lorsque la migration de la phase huile atteint l'état d'équilibre par le fait des forces capillaires, le domaine contaminé est le siège d'un corps d'imprégnation dans lequel le polluant peut être piégé sous forme de gouttelettes isolées et/ou d'amas discontinus dans la zone non saturée et/ou dans l'aquifère saturé formant ainsi une source de contamination à long terme.

Le mécanisme de pollution de notre ressource " eau " débute par le lessivage des constituants de la source de pollution non miscible à l'eau (fig. 1). La conséquence directe de ce lessivage est le relargage des traces dissoutes éventuellement toxiques. Notons que le degré de contamination par ces éléments solubles (notamment le benzène, le toluène, les éthylbenzènes, et les xylènes) est directement lié à la surface de contact entre l'eau et la source de pollution (RAZAKARISOA et al., 1992).

Pour faire face à ce type de contamination, et en comprendre les mécanismes, et pour mettre au point des techniques appropriées de décontamination, la Zone Atelier Franco-Allemande $n^{\circ} 2$ de I'IFARE a conçu et réalisé un dispositif expérimental de grande taille appelé SCERES, Site Contrôlé Expérimental de Recherche pour la Réhabilitation des Eaux et des Sols, bassin étanche de $25 \mathrm{~m}$ de long, $12 \mathrm{~m}$ de large et $3 \mathrm{~m}$ de profondeur. Ce site expérimental permet de reconstituer en vraie grandeur un aquifère alluvial avec nappe à surface libre et de valider, par application directe, des techniques de reconnaissance et des nouveaux procédés de dépollution in situ.

Dans une première partie, le présent article expose la mise en œuvre d'un procédé d'injection de tensioactifs pour décontaminer la partie verticale d'un corps d'imprégnation de gazole sur SCERES. Cet essai a fourni des résultats satisfaisants tant du point de vue du déplacement de l'huile que de la récupération ( $80 \%$ mobilisés, $50 \%$ récupérés). Cependant une réduction significative de la conductivité hydraulique du milieu (chute du débit d'infiltration) a été constatée dans l'heure qui a suivi l'infiltration de la solution tensioactive. 


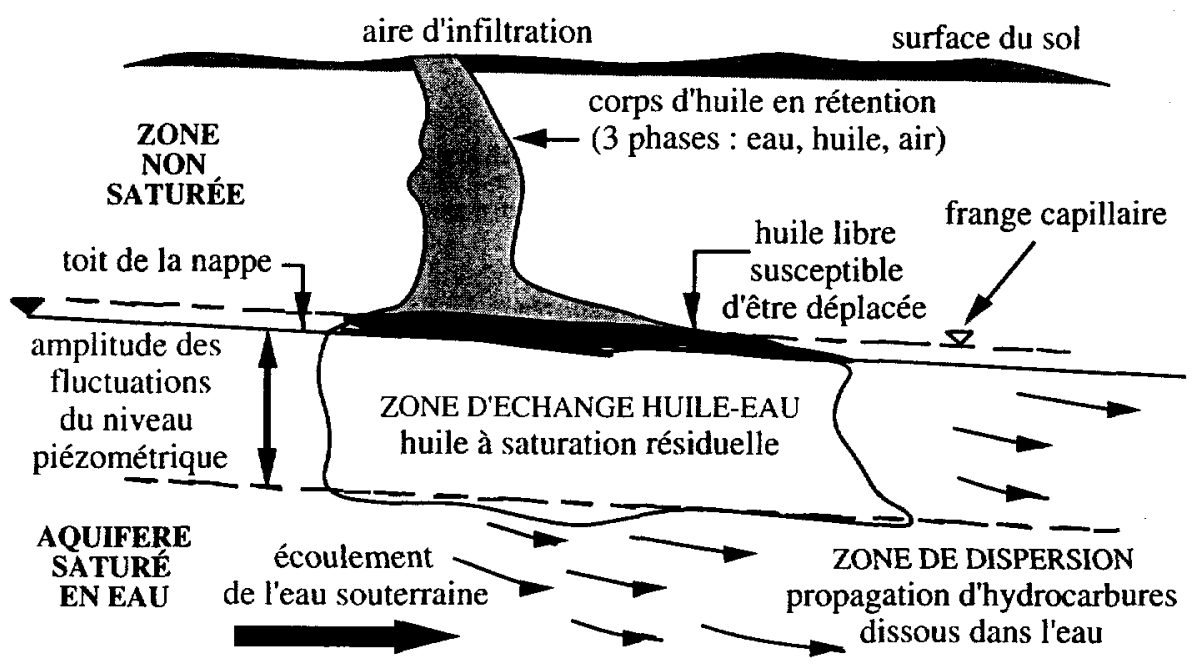

Figure 1 Représentation schématique des mécanismes de pollution d'une nappe alluviale après un déversement accidentel d'hydrocarbures à la surface du sol (d'après RAZAKARISOA et al., 1992).

Schematic representation of the pollution mechanisms of an alluvial aquifer, after an accidental spilling of hydrocarbons.

Plusieurs mécanismes peuvent être à l'origine de ce phénomène. BAVIĖRE et al. (1997) attribuent cette diminution du débit d'infiltration à une mise en solution incomplète des tensioactifs, provoquant ainsi la tendance au colmatage. Dans le but de mieux déterminer l'origine de ce comportement (colmatage du milieu poreux), nous avons mené des essais de percolation de tensioactifs sur des colonnes de milieu poreux en laboratoire.

Dans une deuxième partie, l'article évoque certains arguments pouvant avoir une relation directe avec le colmatage, à partir desquels nous avons fondé nos essais de laboratoire. Les résultats obtenus ont montré que ni le gonflement des argiles dû aux échanges ioniques, ni la précipitation du tensioactif anionique par les ions calcium se substituant au contre ion sodium ne constituent la raison principale de la diminution de la conductivité hydraulique du milieu poreux.

Dans notre cas il apparaît que l'effet de colmatage est induit par la filtration d'agglomérats (cristaux liquides) à l'entrée des modèles physiques (colonnes) utilisés. Cette propriété des tensioactifs a pu être mise en évidence dans les travaux que nous avons effectués au laboratoire.

\section{2 - DESCRIPTION DU SITE}

Le bassin SCERES, construit en béton armé, a été rendu parfaitement étanche par application d'un enduit résistant aux hydrocarbures. Sa structure est enterrée, ce qui contribue à stabiliser la température dans l'aquifère. D'autre part, 
le bassin est couvert d'une bâche pour minimiser les échanges avec l'extérieur (évaporation, lessivage du corps d'imprégnation par les précipitations, etc.). Il est muni aux extrémités, à $50 \mathrm{~cm}$ des parois transversales, de 2 grilles verticales en acier inoxydable délimitant les bacs amont et aval remplis de galets et dans lesquels les niveaux d'eau de référence peuvent être contrôlés (fig. 2). Des équipements adaptés (déversoirs, pompes, cuves, dispositifs de prélèvements et de mesures piézométriques, etc.) sont installés dans deux fosses techniques juxtaposant la structure, et permettent d'assurer la gestion des expérimentations.

Le bassin SCERES est rempli de deux couches de sable siliceux faiblement argileux, un sable principal nommé $\mathrm{H} 2 \mathrm{~F}$ et du sable K10 plus perméable (tabl. 1). La couche de sable grossier K10 d'épaisseur $50 \mathrm{~cm}$ est placée au fond du bassin (fig. 2). Cette couche drainante permet d'appliquer, lors des opérations de décon-

Fosses techniques

(réglage gradient hydraulique, débit, prélèvements d'eau,...)

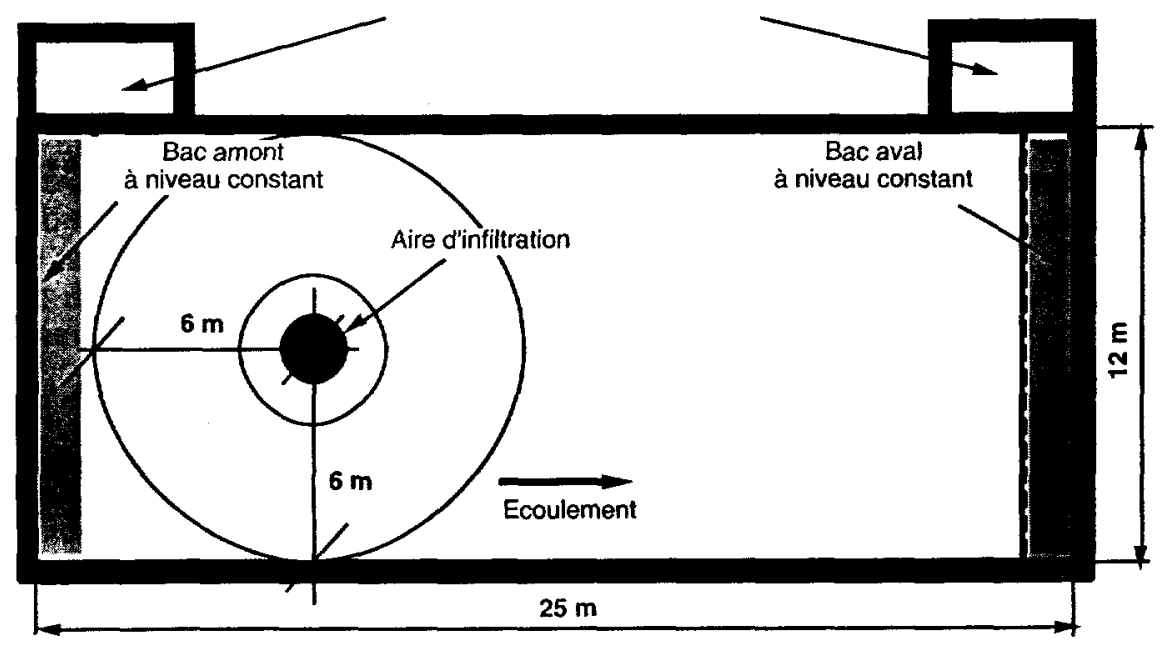

VUE DE DESSUS

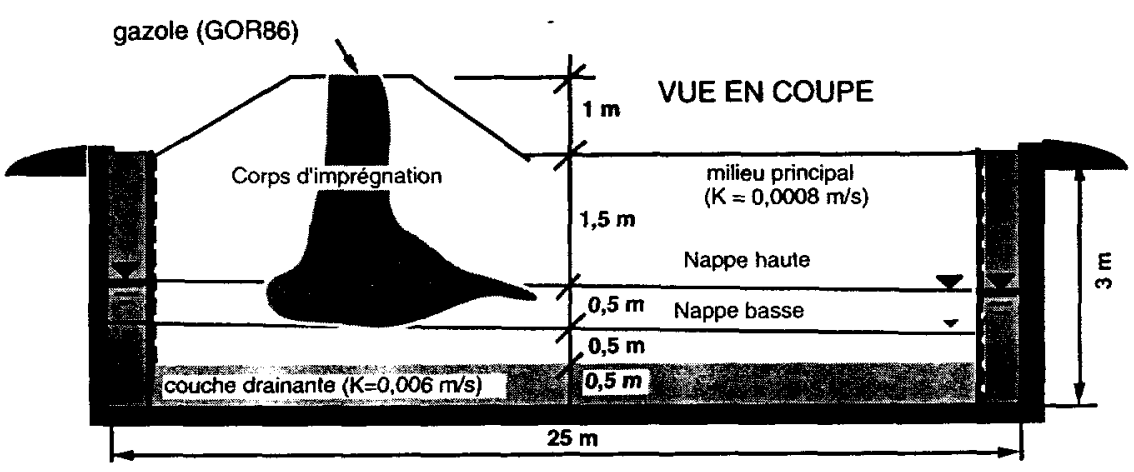

Figure 2 Représentation schématique du dispositif expérimental SCERES (RAZAKARISOA et al., 1995).

Schematic representation of the experimental basin (SCERES). 
Tableau 1 Caractéristiques du système aquifère de l'outil SCERES

Table 1 Characteristics of the SCERES aquifer system

\begin{tabular}{|c|c|c|c|c|c|}
\hline & $\begin{array}{l}\text { Epaisseur } \\
\text { (m) }\end{array}$ & $\underset{(\mathrm{mm})}{d_{50}}$ & $\begin{array}{c}\text { Conductivite } \\
\text { hydraulique } \\
\left(\mathrm{m} . \mathrm{s}^{-1}\right)\end{array}$ & $\begin{array}{c}\text { Porosite } \\
(\%)\end{array}$ & $\begin{array}{l}\text { Frange } \\
\text { capillaire } \\
\text { visuelle } \\
\text { (cm) }\end{array}$ \\
\hline Sable principal (H2F) & 2,5 (dôme : 1) & 0,4 & $8 \times 10^{-4}$ & 40 & $15-20$ \\
\hline \multirow[t]{2}{*}{ Couche drainante (K10) } & 0,5 & 1,1 & $6 \times 10^{-3}$ & 38 & - \\
\hline & & $\begin{array}{l}\text { Épaisseur } \\
\text { moyenne } \\
\text { de la nappe (m) }\end{array}$ & $\begin{array}{l}\text { Débit moyen } \\
\left(m^{3} . \text { heure }{ }^{-1}\right)\end{array}$ & $\begin{array}{l}\text { Température } \\
\text { moyenne } \\
\left.\text { de l'eau ( }{ }^{\circ} \mathrm{C}\right)\end{array}$ & $\begin{array}{c}\text { Gradient } \\
\text { hydraulique } \\
(\%)\end{array}$ \\
\hline \multicolumn{2}{|c|}{ Nappe en position basse (étape 1) } & 1,06 & 0,93 & 11,5 à 15,5 & 0,48 \\
\hline \multicolumn{2}{|c|}{ Nappe en position haute (étape 2) } & 1,53 & 1,07 & 11 à 13,5 & 0,50 \\
\hline \multicolumn{2}{|c|}{ Nappe en position basse (étape 3) } & 1,08 & 1,10 & 13 à 16 & 0,54 \\
\hline
\end{tabular}

tamination, des débits de pompage suffisants sans être gênés par l'influence des parois. Un dôme de sable de $1 \mathrm{~m}$ de hauteur est adopté dans le but de disposer d'une profondeur plus importante de zone non saturée (fig. 2).

Après l'établissement de l'état d'équilibre du système aquifère (niveau piézométrique, débit, etc.), une source de pollution a été créée par déversement d'un gazole routier dénommé GOR 86 (densité $=0,84$; viscosité $=3,91 \mathrm{mPa} .5$ ). Le produit GOR 86 est représentatif de plusieurs cas de pollution de nappe par des produits pétroliers et contient une large gamme d'hydrocarbures aromatiques et aliphatiques.

Le 21 juillet 1993, 482 litres de gazole sont déversés au-dessus du dôme de sable du bassin, sur une aire de $1,50 \mathrm{~m}$ de diamètre, centrée à $6 \mathrm{~m}$ des parois amont et latérales et située à une altitude de 2,90 m au-dessus de la surface libre de la nappe (fig. 2). Le contrôle et la régulation du débit d'infiltration ont été assurés grâce à la mise en place d'un filtre de ralentissement conçu pour cet effet. Six litres du produit ont été retenus dans ce dispositif après le déversement. Le débit d'infiltration de la phase huile, sous charge constante, à travers le filtre, est de 9 litres par minute.

\section{3 - ÉVALUATION DU DEGRÉ ET DE L'ÉTENDUE DE LA CONTAMINATION}

Le suivi et la reconnaissance de la contamination du système aquifère ont été conduits selon 3 étapes (fig. 3) simulant un battement de nappe :

- Infiltration de 476 litres du polluant GOR 86 et migration de la phase huile jusqu'à l'établissement de l'état d'équilibre (du 21 juillet 1993 au 9 décembre 1993).

- Élévation du niveau piézométrique de la nappe de $50 \mathrm{~cm}$ (du 10 décembre 1993 au 27 mars 1994).

- Rabaissement de $50 \mathrm{~cm}$ de la surface libre de la nappe avant le démarrage des expériences de décontamination (depuis le 28 mars 1994). 


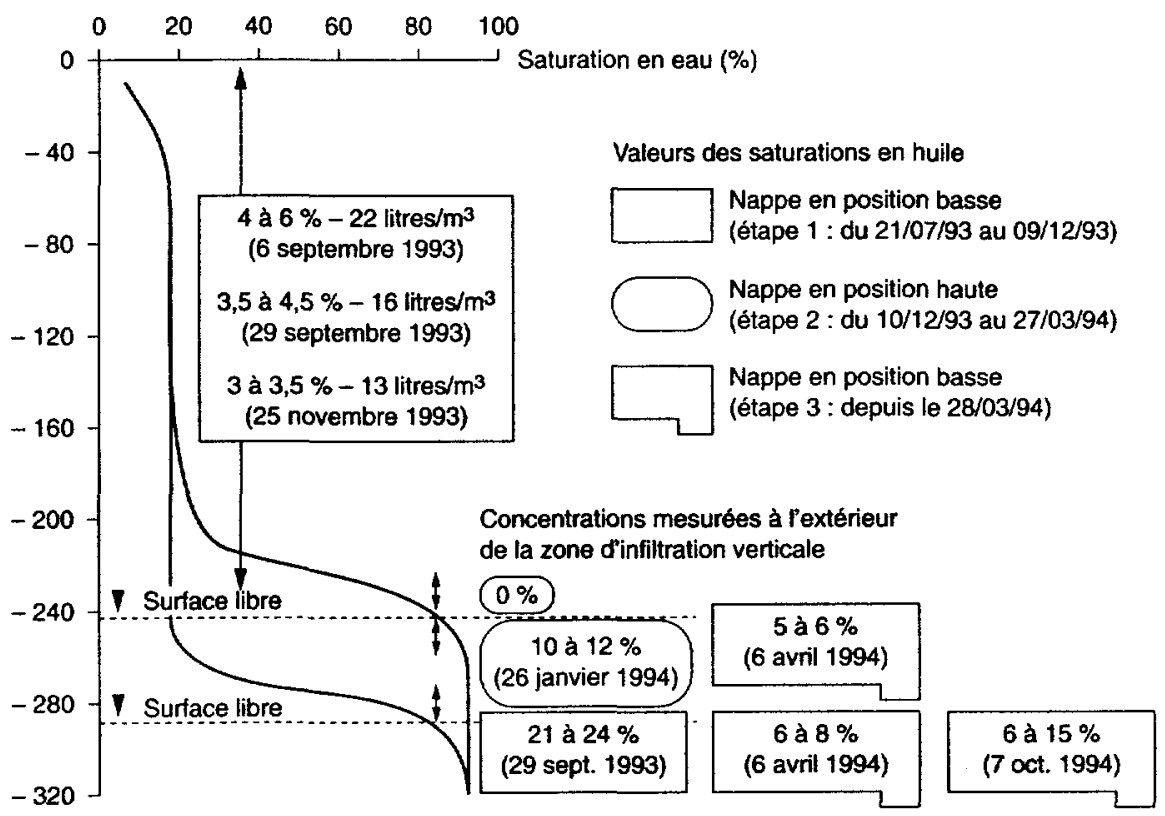

Profondeur (cm)

Figure 3 Profils hydriques dans le bassin SCERES (mesure par sonde à neutron) et évolution des saturations en huile du corps d'imprégnation (RAZAKARISOA et al., 1995).

Water saturation profile (determined by moisture gauge SOLO 40 with neutron probe) and oil saturation measurements after core-drills investigations.

Dans le but de détecter et quantifier la pollution du sous-sol, et pour obtenir une détermination précise de l'étendue de la pollution, nous avons conçu et mis en œuvre une sonde de prélèvement non destructive ayant la forme d'une tige de $3 \mathrm{~m}$ de long et de $2 \mathrm{~cm}$ de diamètre. À l'aide de cette sonde, de faibles quantités de sable (10 à $12 \mathrm{~g}$ ) sont prélevées localement en des points bien définis dans les zones non saturée et saturée sans altérer les échantillons pendant leur passage à travers le sous-sol, lors du retrait de la sonde. La phase huile présente dans les échantillons de sable est extraite au tétrachlorure de carbone et les concentrations en gazole sont déterminées par spectrophotométrie infrarouge.

Après le déversement du polluant, les mesures par échantillonnage de sable ont montré que la migration du GOR 86 s'effectue jusqu'au toit de la frange capillaire sans atteindre complètement la surface libre de la nappe et laisse dans la zone non saturée un domaine imprégné de forme cylindrique de hauteur $2,75 \mathrm{~m}$ et de diamètre compris entre $1,80 \mathrm{~m}$ et $1,85 \mathrm{~m}$. La saturation résiduelle en polluant dans cette zone est de 3 à $4 \%$. La migration de la phase huile est très lente : les différentes campagnes de prélèvement ont montré que la stabilisation des valeurs de saturation en huile est obtenue après une période de 4 mois.

L'extension horizontale du polluant au niveau de la frange capillaire se concentre sur une aire circulaire de $5,60 \mathrm{~m}$ à $5,90 \mathrm{~m}$ de diamètre et une couche de l'ordre de $10 \mathrm{~cm}$ d'épaisseur (saturation élevée de 21 à $24 \%$, voir fig. 3). 


\section{4 - MÉTHODE DE DÉCONTAMINATION IN SITU}

Au centre de l'aire d'infiltration, un puits de diamètre $20 \mathrm{~cm}$ a été foré pour permettre l'application des procédés de décontamination (fig. 4). À l'intérieur de ce puits sont installées deux pompes : l'une, immergée au fond, permet de créer un cône de dépression (rabattement de nappe variant entre 6 et $10 \mathrm{~cm}$ ), l'autre permet de récupérer par écrémage le gazole en phase à la surface de la nappe. Le pompage adopté (débit variant de 0,7 à $1,2 \mathrm{~m}^{3} / \mathrm{h}$ ), maintenu pendant 58 jours, a permis de récupérer 110 litres de gazole, soit $23 \%$ du volume infiltré.

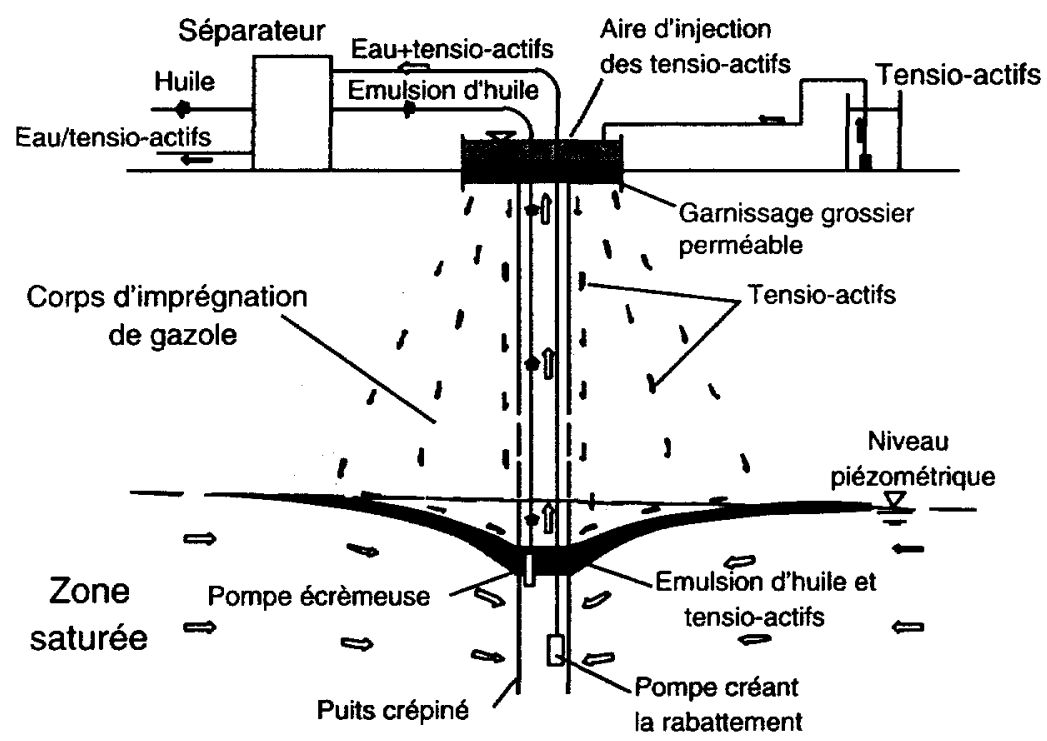

Figure 4 Schéma du principe d'injection de tensioactifs dans le bassin SCERES (VAN DORPE et al., 1995).

Schematic representation of the surfactant injection on the SCERES basin.

Un essai de ventilation par création d'une dépression d'air (12 mbars) dans le puits a été effectué après le pompage de l'huile en phase, en gardant le même rabattement. Le but de cette aspiration d'air au sein du milieu poreux est de provoquer une migration éventuelle de l'huile résiduelle vers le puits. Cette opération a permis de récupérer une douzaine de litres de gazole supplémentaires.

Suite à cette opération de récupération de l'huile mobile surnageant dans la frange capillaire, une infiltration de tensioactifs a été appliquée depuis la surface afin de nettoyer la zone non saturée contenant le gazole résiduel piégé (fig. 4). Le rabattement adopté est plus important $(\mathrm{h}=17 \mathrm{~cm})$. Les tensioactifs, formulés et fournis par l'Institut Français du Pétrole, ont été infiltrés sous charge constante sur une aire de diamètre $1,70 \mathrm{~m}$ au sommet du dôme. La solution de tensioactifs mise en œuvre (appellation RESOL 30) est un mélange d'un tensioactif anionique et d'un tensioactif non ionique dilués dans l'eau du réseau à raison de $5 \mathrm{~g} / \mathrm{l}$ chacun auquel on ajoute du chlorure de sodium à raison de $1,5 \mathrm{~g} /$. Dans cette solution aqueuse, le tensioactif non ionique améliore la solubilisation de l'anioni- 
que et la concentration en sel est ajustée pour optimiser le comportement de phases du système Eau/Gazole/Tensioactifs.

Au préalable, le milieu a été saturé en eau (infiltration de $19 \mathrm{~m}^{3}$ ) afin de respecter les conditions optimales définies par les expériences de laboratoire (DUCREUX et al., 1995). Au total $23 \mathrm{~m}^{3}$ de solution de tensioactifs sont infiltrés malgré des problèmes de colmatage du milieu se traduisant par une chute du débit d'infiltration (fig. 5).

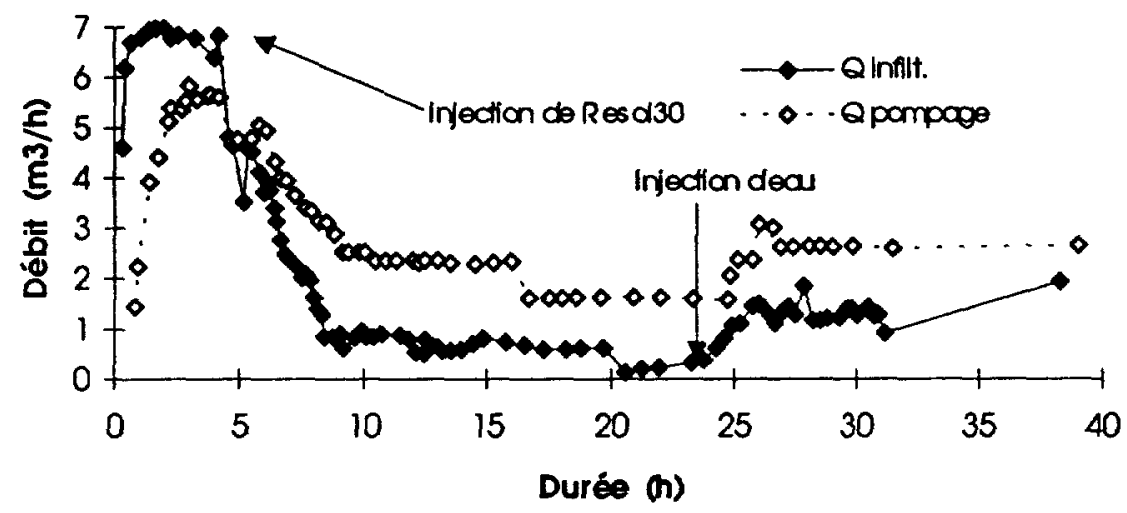

Figure 5 Évolution du débit d'infiltration du RESOL 30 dans SCERES.

Evolution of the infiltration flow rate of the RESOL 30 in SCERES.

Lors de l'infiltration préliminaire d'eau (pour la saturation du milieu), le débit s'est maintenu entre 6,5 et $7 \mathrm{~m}^{3} / \mathrm{h}$ pendant près de 4 heures. Cette valeur est proche de la valeur théorique du débit d'infiltration maximum, $Q_{\text {imax }}$ dont l'écart peut s'expliquer par la présence de gazole résiduel qui a pour effet de réduire la conductivité hydraulique du milieu, en supposant que l'infiltration se fait à travers un disque de $1,7 \mathrm{~m}$ de diamètre, avec $\mathrm{K}=8 \cdot 10^{-4} \mathrm{~m} / \mathrm{s}, \mathrm{Q}_{\mathrm{imax}}=7,3 \mathrm{~m}^{3} / \mathrm{h}$.

Dès le début de l'injection du RESOL 30 et pendant le passage des 12 premiers mètres cubes, le débit d'infiltration chute régulièrement. Ensuite, le milieu se colmate progressivement jusqu'à annulation du débit d'infiltration (fig. 5). Le débit de pompage, double de celui de l'injection, a permis de récupérer de façon satisfaisante la solution injectée (bilan de plus de $95 \%$ ) et de récupérer 58 litres de gazole dont $43 \%$ sous forme décantable et le reste sous forme émulsionnée dans la solution. Sur la base de la quantité d'huile résiduelle de la zone d'infiltration verticale (estimée à 121 litres), le taux de récupération est de près de $50 \%$ (fig. 6).

Le lessivage par l'eau douce, qui intervient après l'infiltration de $23 \mathrm{~m}^{3}$ de RESOL 30 , permet de retrouver un débit de $1,5 \mathrm{~m}^{3} / \mathrm{h}$ après passage de $3 \mathrm{~m}^{3}$ d'eau. Ce débit n'évolue pas sensiblement, malgré l'injection de $17 \mathrm{~m}^{3}$ d'eau supplémentaires.

Cette réduction de la conductivité hydraulique du milieu poreux est souvent expliquée par une précipitation des tensioactifs, notamment anioniques, en présence des ions calcium, se substituant au sodium, ici le contre ion du tensioactif anionique (ALLRED et BROWN, 1994 ; CELIK et al., 1982). D'autres auteurs (ZUN- 


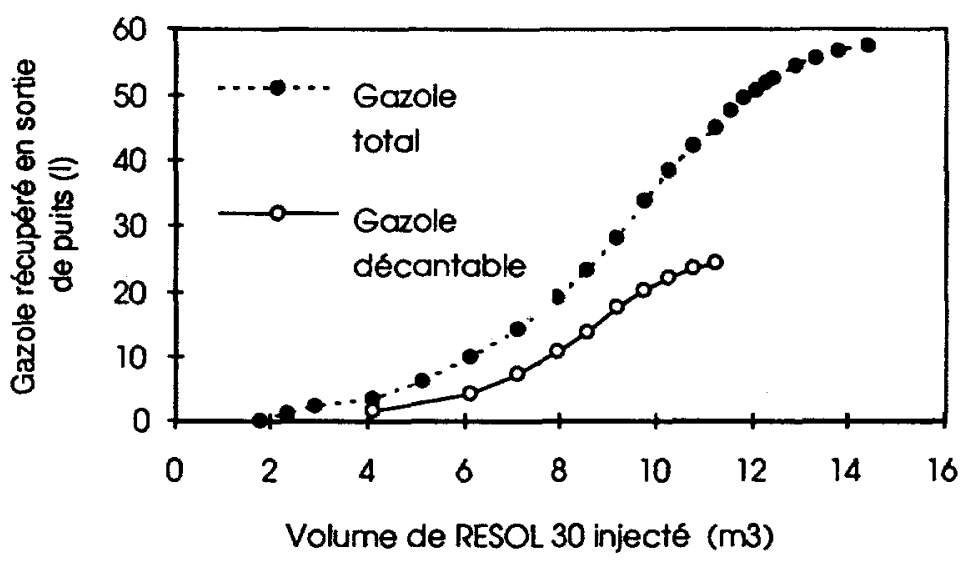

Figure 6 Récupération du gazole de la zone d'infiltration verticale (VAN DORPE et al., 1995).

Cumulative volume of oil recovered by the infiltration of the surfactants.

DEL et SIFFERT, 1984) imputent ce phénomène à la seule présence des argiles. Une troisième hypothèse serait que les tensioactifs à des concentrations relativement importantes auraient tendance à s'agglomérer pour former des objets (cristaux liquides) de taille importante comparée à celle des micelles mixtes. En vue de vérifier ces hypothèses au laboratoire, nous avons réalisé des essais de percolation de tensioactifs dans des colonnes remplies de sable $\mathrm{H} 2 \mathrm{~F}$ provenant du bassin expérimental.

\section{5 - APPROCHE EXPÉRIMENTALE AU LABORATOIRE}

\subsection{Mise en évidence du colmatage de la matrice}

Les essais de percolation sont menés sur des colonnes $\mathrm{C} 1$ et $\mathrm{C} 2$ en Plexiglas (C1 : hauteur $150 \mathrm{~cm}$, diamètre $10 \mathrm{~cm}$; $\mathrm{C}_{2}$ : hauteur $40 \mathrm{~cm}$, diamètre $5 \mathrm{~cm}$ ) remplies de sable et munies de tubes manométriques pour suivre l'évolution de la conductivité hydraulique dans les différentes parties de la colonne. Les caractéristiques du modèle physique sont indiquées sur la figure 7 .

Le phénomène de colmatage (forte diminution de la conductivité hydraulique K) est mis en évidence dans la zone supérieure de la colonne tandis que la partie restante ne semble pas être particulièrement touchée par cette réduction de la perméabilité (fig. 8). Si ce phénomène de colmatage était dû à la précipitation des tensioactifs anioniques sous forme calcique (le calcium provenant des échanges ioniques avec les argiles du sable) (ALLRED et BROWN, 1994), nous aurions observé une diminution de la conductivité hydraulique sur la totalité de la matrice poreuse. D'après les résultats de la figure 8 , il apparaît que c'est une éventuelle filtration des tensioactifs par des particules fines (argiles) qui se mani- 


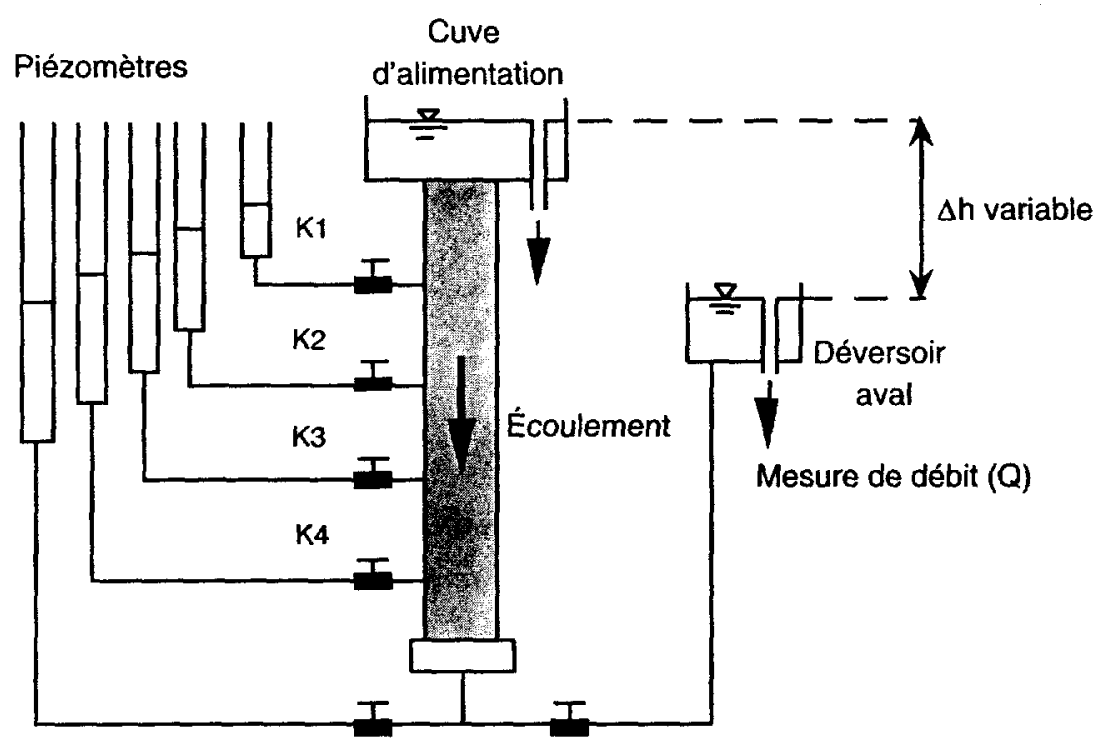

Figure 7 Schéma du dispositif expérimental réalisé au laboratoire.

Scheme of the experimental setup.

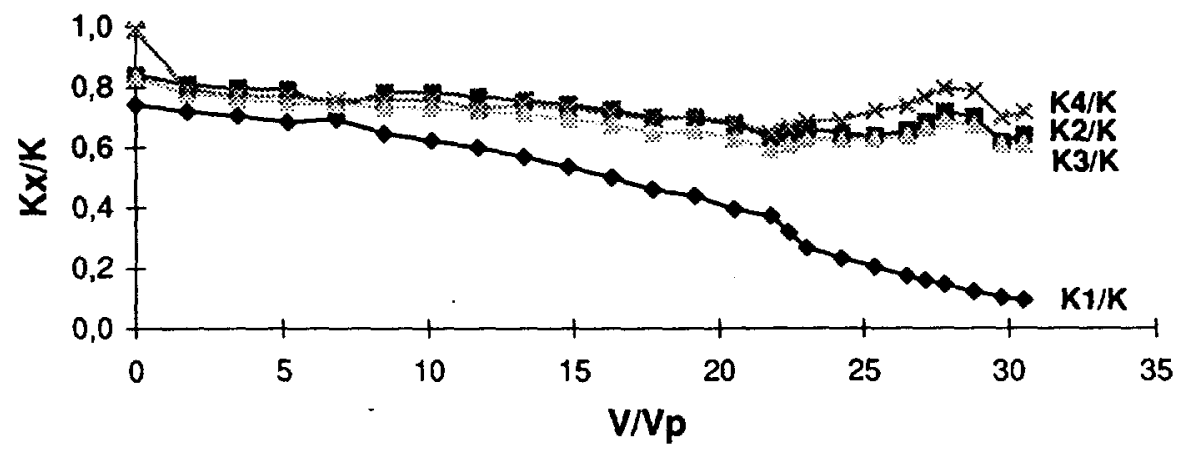

Figure 8 Localisation du colmatage dans les essais menés sur colonnes.

Location of the plugging.

feste dans la partie haute de la colonne et qui provoque le colmatage après qu'un certain volume (correspondant à $V N p \cong 7 ; V=$ volume écoulé $; \mathrm{V}=$ volume de pores du milieu) de tensioactifs ait été injecté.

La vérification du rôle des particules fines est montrée sur la figure 9 dans laquelle les résultats des essais menés sans la présence des fines ont été obtenus en procédant à une peptisation des particules argileuses contenues dans le milieu (BEHRA, 1987). Le phénomène de colmatage persiste malgré l'élimination des fines dans la matrice poreuse, ce qui nous conduit à écarter la responsabilité des particules argileuses dans l'effet de diminution du débit d'écoulement $(Q)$ de 


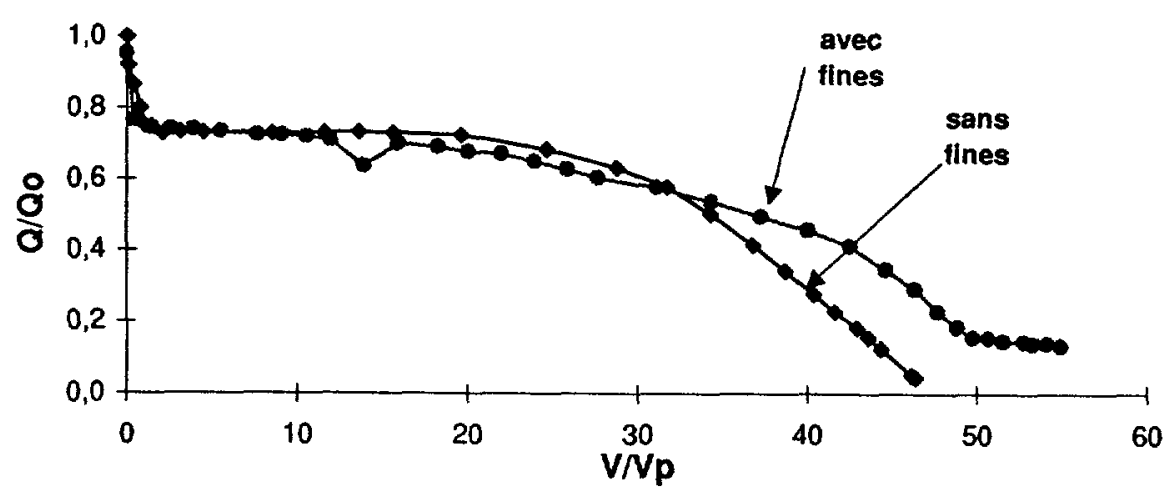

Figure 9 Effet des particules argileuses sur l'infiltration du RESOL 30.

Effect of clay minerals on the surfactant flow.

la solution de tensioactifs en milieu poreux et à considérer que les propriétés mêmes des solutions de tensioactifs utilisés jouent un rôle dans ce processus. II est à noter que la première chute du rapport du débit $Q / Q 0(1,0$ à 0,8$)$ sur les figures $9,10,11$ et 12 est due à la différence de viscosité qui existe entre l'eau ayant servi à la saturation préalable du milieu poreux et la solution tensioactive (facteur 1,5).

L'effet du gradient hydraulique sur l'infiltration du RESOL 30 est également étudié et les résultats sont illustrés par la figure 10. Le colmatage se produit après un volume injecté d'autant plus grand que le gradient hydraulique est plus élevé (fig. 10).

En fait, la tendance au colmatage dépend du temps: pour tous les essais effectués, une diminution significative du débit est ressentie au-delà de 10 heures jusqu'à son annulation au bout de 24 heures (fig. 11). Cette notion de cinétique qui intervient dans la décroissance du débit d'infiltration nous a incité à examiner les propriétés du RESOL 30 .

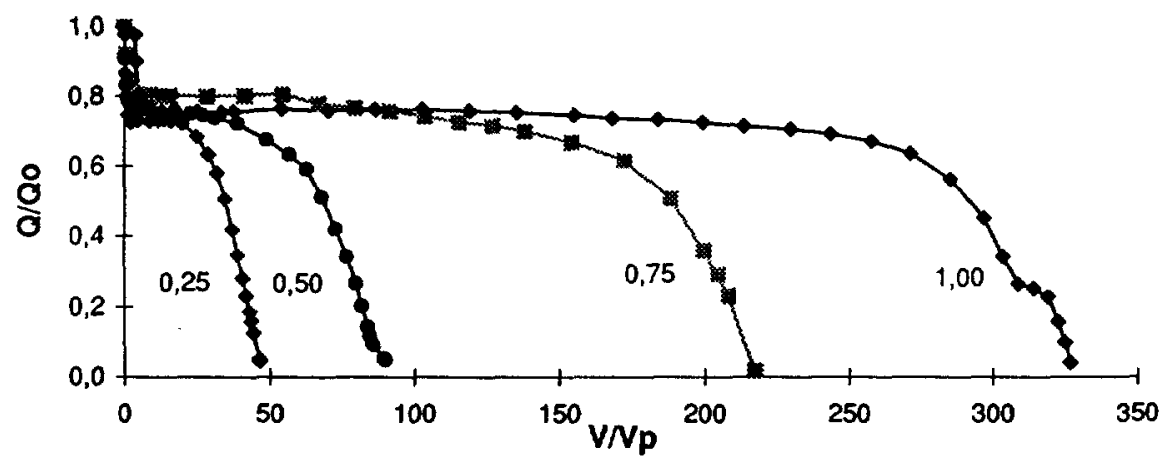

Figure 10 Effet du gradient hydraulique sur l'infiltration du RESOL 30.

Effect of the hydraulic gradient on the RESOL 30 infiltration. 


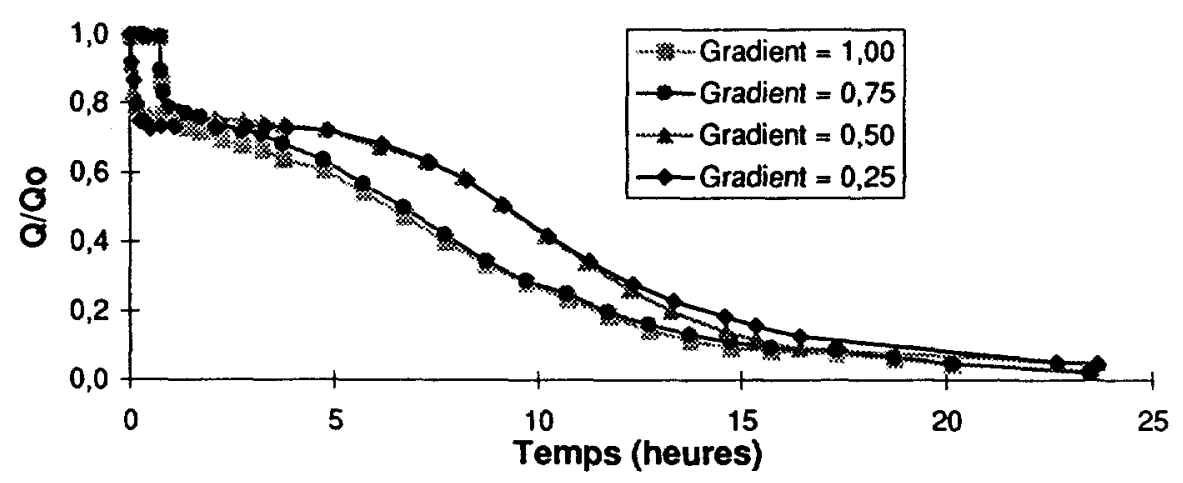

Figure 11 Évolution du débit d'infiltration du RESOL 30 en fonction du temps.

Evolution of the RESOL 30 flowrate vs time.

Cet aspect est appréhendé en modifiant le temps de vieillissement de la solution avant l'injection, ce qui a permis d'exploiter les résultats de trois essais conduits de la façon suivante : un premier essai de percolation effectué avec une solution « fraîchement » préparée, un second avec une solution mise au repos pendant 24 heures et un troisième essai effectué avec une solution ayant séjourné 52 heures au laboratoire, à gradient hydraulique constant pour chaque essai $(0,2)$. Les résultats illustrés par la figure 12 mettent en évidence les modifications des caractéristiques du RESOL 30 dans le temps, autrement dit la solution de tensioactifs ne doit pas séjourner trop longtemps avant l'essai de percolation pour conserver une injectabilité acceptable.

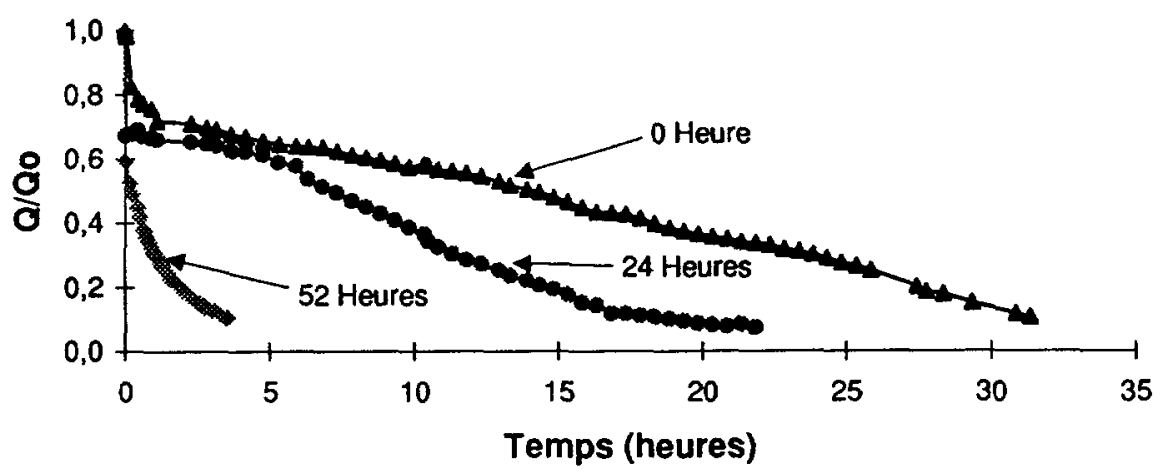

Figure 12 Stabilité de la solution de RESOL 30 en fonction du temps.

Stability of the RESOL 30 solution vs time.

\subsection{Interprétation des phénomènes rencontrés}

L'ensemble des expériences visant à appréhender le comportement du RESOL 30 lors de son passage en milieu poreux (sable siliceux faiblement argileux) ont montré que la précipitation du tensioactif anionique ne pouvait à elle 
seule occasionner le phénomène de colmatage. En effet, les ions calcium présents dans la matrice peuvent provoquer cette précipitation et cela se traduirait par une réduction de la conductivité hydraulique dans tout le milieu (les échanges ne pouvant se limiter à la seule partie située à l'entrée du dispositif expérimental). Il a été montré qu'une certaine instabilité du mélange de tensioactifs se produit. L'essai effectué avec un gradient hydraulique élevé permet, certes, d'injecter une quantité plus importante, mais n'évite pas le colmatage qui, dans tous les cas de figure, se manifeste au bout de 24 heures d'infiltration. Par ailleurs, une solution de RESOL 30 qui est laissée au repos longtemps avant son utilisation engendre un rapide colmatage du milieu poreux.

Le principal facteur responsable du phénomène de colmatage est dû ici à la seule solution de RESOL 30 indépendamment des interactions qui peuvent avoir lieu entre la solution aqueuse et la matrice poreuse (adsorption, précipitation, etc.). De plus, la filtration d'objets dispersés dans le milieu aqueux, nous amène à supposer que le RESOL 30 a tendance à former des agrégats, qui après un certain temps deviennent suffisamment volumineux pour être retenus à la surface de la matrice poreuse et provoquer le colmatage. Dans notre cas, la concentration de la solution de tensioactifs $(10 \mathrm{~g} /$ ) est relativement importante, de plus la présence des ions calcium dans l'eau de préparation facilite sa tendance à s'agglomérer pour former des systèmes lamellaires, cylindriques ou mixtes (Buhler, 1996). En ce qui concerne le RESOL 30, l'hypothèse retenue est la formation de cristaux liquides mixtes (fig. 13) que nous avons observés lors des mesures de granulométrie par diffusion laser effectuées à l'Institut Français du Pétrole. Il apparaît que la taille moyenne des objets qui diffusent est de l'ordre de $450 \mathrm{~nm}$ soit près de 10 à 20 fois supérieure à la taille moyenne d'une micelle mixte (30 à $40 \mathrm{~nm}$ ).

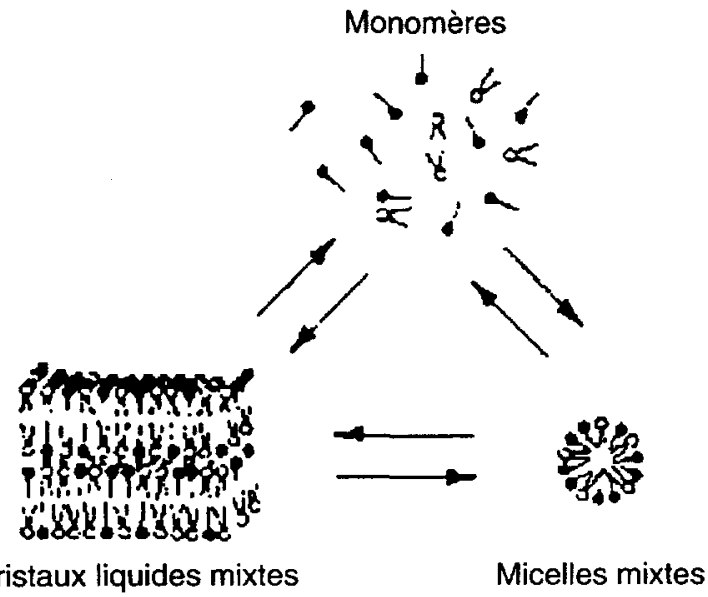

Cristaux liquides mixtes

Micelles mixtes

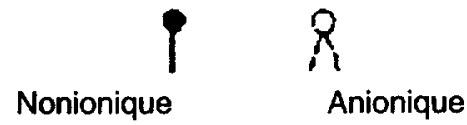

Figure 13 Schéma des équilibres monomères - micelles mixtes - cristaux liquides (BEAUVAIS, 1989).

Schematic representation of equilibrium monomeres - micelles - cristals. 


\section{6 - CONCLUSIONS ET PERSPECTIVES}

L'infiltration du mélange de tensioactifs (le RESOL 30) depuis la surface du dôme de SCERES pour décontaminer la zone verticale du corps d'imprégnation formé de gazole a été conduite dans le but d'évaluer l'efficacité de ce procédé lors de son application pour une réhabilitation in situ. Les résultats de cet essai de dépollution ont permis, d'une part de mettre en évidence sur le terrain les possibilités des tensioactifs à remobiliser et déplacer le polluant piégé à saturation résiduelle, d'autre part de montrer les difficultés éventuelles liées au comportement hydrodynamique des tensioactifs se traduisant par la réduction de la conductivité hydraulique du milieu traversé et entraînant la quasi-annulation du débit d'infiltration. L'approche expérimentale en laboratoire à l'aide d'essais de percolation de tensioactifs sur colonnes de milieu poreux a permis d'appréhender certains comportements de la solution de tensioactifs, de mettre en évidence le colmatage de la matrice poreuse et d'expliquer l'origine possible de ce phénomène.

De cette étude, il est apparu que la réduction de la conductivité hydraulique affectait dans tous les cas de figure la partie amont du modèle physique du fait de l'instabilité de la solution de tensioactifs utilisée, celle-ci se traduisant par la tendance des micelles mixtes à s'agglomérer sous l'effet des ions calcium présents dans l'eau de préparation. Cette agglomération conduit à la formation d'objets (cristaux liquides) de taille suffisamment grande pour être retenus à l'entrée du milieu poreux par simple filtration. Le phénomène de colmatage peut jouer un rôle important sur le plan de la récupération car son effet peut conduire à une insuffisance de gradient de pression au voisinage du puits de pompage.

La poursuite des recherches menées au sein du laboratoire vise d'une part à améliorer la mise en solution du mélange tensioactif, d'autre part à disperser les micelles pour atténuer l'effet d'agglomération des tensioactifs et augmenter leur injectabilité.

\section{REMERCIEMENTS}

Cette recherche conduite à l'IFARE a été menée dans le cadre des activité de la Zone Atelier Franco-Allemande (ZAFA) “ Protection des aquifères contre la pollution par les hydrocarbures et dérivés ", et a bénéficié du financement du Contrat de Plan Etat (CNRS - Programme Environnement - SPI, Ministère de l'Environnement, ULP) et Région Alsace. La zone Atelier regroupe les universités de Strasbourg, de Stuttgart, de Karlsruhe, de Tübingen et Dresden, ainsi que I'Institut Français du Pétrole, le Burgéap et Anjou Recherche de la Société Générale des Eaux. Cette ZAFA est animée par le Laboratoire d'Hydrodynamique des Milieux Poreux de l'Institut de Mécanique des Fluides de Strasbourg. 


\section{RÉFÉRENCES BIBLIOGRAPHIQUES}

ALLRED, B., BROWN, G. (1994). Surfactantinduced reductions in soil hydraulic conductivity. GWMR, Spring 1994, 174-184.

BAVIERE, M., BOCARD, C., DUCREUX, J., MONIN, M., MUNTZER, P., RAZAKARISOA, O. (1997). In situ Remediation of Oil-Contaminated Soils with Surfactants : Laboratory and Pilot Studies. Paper SPE 37258, Proceedings, International Symposium on Oilfield Chemistry. Houston, Texas, 18-21/02/1997, 481-491.

BEAUVAIS, G. (1989). Propagation d'un mélange de tensioactifs anionique et nonionique dans un milieu poreux adsorbant. Thèse de Doctorat de l'Université Paris VI en Sciences Pétrolières.

BEHRA, P. (1987). Etude du comportement d'un micropolluant métallique -le mercureau cours de sa migration à travers un milieu poreux saturé : Identification expérimentale des mécanismes d'échanges et modélisation des phénomènes. Thèse de Doctorat, Université Louis Pasteur de Strasbourg.

BUHLER, E. (1996). Etude par diffusion du rayonnement des propriétés structurales et dynamiques de systèmes autoassemblés de tensioactifs. Thèse de Doctorat, Université Louis Pasteur de Strasbourg.

CELIK, M.S., MANEV, E.D., SOMASUNDARAN, P. (1982). Sulfonate precipitationredissolution-reprecipitation in inorganic electrolytes. Proceedings of the American Institute of Chemical Engineers Symposium, 78(212), 86-96.
DUCREUX, J., BAVIERE, M., SEABRA, P., RAZAKARISOA, O., SCHÄFER, G., ARNAUD, C. (1995). Surfactant-Aided Recovery. In situ Bioremdiation Process for Oil Contaminated Sites. Proceedings of Third International Symposium « In situ and On-Site Bioreclamation ", San Diego, California, 24-27 avril 1995, 435-443.

RAZAKARISOA, O., MUNTZER, P., RIMMELIN, P., ZILLIOX, L. (1992). Incidence de la source de pollution sur la dissolution et la rétention sélective d'hydrocarbures en milieu poreux saturé en eau. Revue des Sciences de l'Eau, 5(2), 157-178.

RAZAKARISOA, O., MUNTZER, P., SCHÄFER, G., ZILLIOX, L., DUCREUX, J., ARNAUD, C. (1995). Pollution contrôlée d'aquifère alluvial par infiltration de gazole: impact du battement de nappe sur le degré de contamination de l'eau souterraine. Proceedings of the International Conference on "Groundwater Quality: Remediation and Protection ", Prague, Publication IAHS, $n^{\circ} 225,139-147$.

VAN DORPE, F., BETTAHAR, M., OTT, C., RAZAKARISOA, O. (1995). Pollution contrôlée d'aquifère alluvial par infiltration de gazole sur site expérimental - Décontamination hydraulique et drainage activé par tensioactifs. Actes du $12^{e}$ Congrès Français de Mécanique, AUM Eds, Strasbourg 1995, 85-88.

ZUNDEL, J.P., SIFFERT, B. (1984). Mécanisme de rétention de l'octylbenzènesulfonate de sodium sur les minéraux argileux. Actes du colloques et séminaires (42), Technip Eds, Nancy 1985, 447-462. 